\title{
Direct DNA Sequencing-Based Analysis of Microbiota Associated with Hematological Malignancies in the Eastern Province of Saudi Arabia
}

\author{
Faisal M. Alzahrani $\left(\mathbb{D},{ }^{1}\right.$ Ali Al-Amri, ${ }^{2}$ Saeed Sattar Shaikh $\left(\mathbb{D},{ }^{1}\right.$ Muzaheed $\mathbb{D},{ }^{1}$ \\ Amer Ibrahim Alomar $\mathbb{D}^{1},{ }^{1}$ Sadananda Acharya $\mathbb{D}^{0},{ }^{3}$ Maryam Ahmed Aldossary, \\ and Fathelrahman Mahdi Hassan $\left.{ }^{1}\right)^{1}$ \\ ${ }^{1}$ Department of Clinical Laboratory Science, College of Applied Medical Sciences, Imam Abdulrahman Bin Faisal University, \\ Dammam, Saudi Arabia \\ ${ }^{2}$ Department of Internal Medicine/Oncology, College of Medicine, King Fahd Hospital of the Imam Abdulrahman Bin \\ Faisal University, Dammam, Saudi Arabia \\ ${ }^{3}$ Department of Public Health, College of Public Health, Imam Abdulrahman Bin Faisal University, Dammam, Saudi Arabia
}

Correspondence should be addressed to Saeed Sattar Shaikh; ssshaikh@iau.edu.sa

Received 23 August 2020; Accepted 25 January 2021; Published 3 February 2021

Academic Editor: Hao Chen

Copyright (c) 2021 Faisal M. Alzahrani et al. This is an open access article distributed under the Creative Commons Attribution License, which permits unrestricted use, distribution, and reproduction in any medium, provided the original work is properly cited.

\begin{abstract}
Introduction. Bloodstream infections (BSI) among patients with hematological malignancies (HM) could predispose them to higher morbidity and mortality for various underlying conditions. Several microorganisms, either pathogenic or opportunistic normal human flora, could cause severe bacteremia and septicemia. While conventional methods have their own limitations, molecular methods such as next-generation sequencing (NGS) can detect these blood infections with more reliability, specificity, and sensitivity, in addition to information on microbial population landscape. Methodology. Blood samples from HM patients $(n=50)$ and volunteer blood donor control individuals with no HM $(n=50)$ were subjected to $16 \mathrm{~S}$ rRA gene amplification using standard PCR protocols. A metagenomic library was prepared, and NGS was run on a MiSeq (Illumina) sequencer. Sequence reads were analyzed using MiSeq Reporter, and microbial taxa were aligned using the Green Genes library. Results. $82 \%$ of the patients showed BSI with Gram-negative bacteria as the most predominant group. E. coli comprised a major chunk of the bacterial population (19.51\%), followed by K. pneumoniae (17.07\%). The CoNS and Viridans Streptococci groups are $17.07 \%$ and $14.63 \%$, respectively. Other major species were S. aureus (9.75\%), P. aeruginosa (7.31\%), A. baumannii (4.87\%), E. cloacae (4.87\%), and P. mirabilis (4.87\%). $34.14 \%$ of the cases among patients showed a Gram-positive infection, while $14.63 \%$ showed polymicrobial infections. Conclusion. Most of the BSI in patients were characterized by polymicrobial infections, unlike the control samples. Molecular methods like NGS showed robust, fast, and specific identification of infectious agents in BSI in $\mathrm{HM}$, indicating the possibility of its application in routine follow-up of such patients for infections.
\end{abstract}

\section{Introduction}

Hematological malignancies are cancers of the bloodproducing cells. Hematopoietic stem cells undergo a highly regulated and coordinated differentiation and maturation process to produce various blood cells. An aberration in the regulatory pathway confers the cells a neoplastic characteristic clinically presenting as hematological malignancy. Hema- tological malignancies are various heterogenous conditions resulting from abnormal cell division and proliferation in the bone marrow and the lymphatic system [1]. Hematological malignancies are the $5^{\text {th }}$ most common causes of cancers in Saudi Arabia [2]. Yearly, approximately over a thousand people die from this disease in the country (https://www .who.int). This becomes nearly $10 \%$ of deaths due to various cancers. Hematological malignancies include acute and 
chronic myeloid leukemias, acute and chronic lymphocytic leukemias, and various lymphomas and myelomas. Among cancer patients, particularly with hematological malignancies such as leukemias and lymphomas, bacteremia and bacterial septicemia are a major cause of poor patient outcome [3]. Patients with hematological malignancies have higher risks of contracting bloodstream infections (BSI) owing to immunological dysfunction as a result of chemo and radiotherapy. BSI can be caused by various opportunistic bacteria, viruses, and fungi. Microbial invasion and growth in the bloodstream releases toxins characterized by life-threatening multiorgan failure. Bacterial septicemia is one of the top ten causes of death worldwide and more so in patients with hematological malignancies. Successful management of hematological malignancies includes the proper study of local infections, complications, and antibiotic resistance patterns among the isolated microbial pathogens. However, rapid, reliable, and specific microbial detection methods are limited for the accurate diagnosis of infectious etiology. Conventional culturebased methods take a prolonged time duration and are often challenged with specificity and sensitivity issues. Molecular detection methods such as polymerase chain reaction (PCR), though, are quite popular, and they are associated with problems in interpretation, accuracy, and requirement for high technical inputs. Of late, deoxyribonucleic acid (DNA) sequencing methods have been used widely to deduce the genetic information and identify the causative microorganisms. Next-generation sequencing (NGS) has been very popular for its robustness, speed, accuracy, and large number of samples it can handle at a time. This technology can identify viable, dead, viable but not culturable (VBNC) bacteria, fungi, and viruses in multiple heterogeneous sample pools. Additionally, the NGS sequence can give us information on the microbiome-the complete microbial population structure of the study subject unraveling a plethora of information required for successful treatment, including the antibiotic resistance genes that the organism carries.

Management of suspected BSIs with hematological malignancies with information derived from NGS sequencing can pave the way for improvement in patient care. Microbial infections in patients with hematological malignancies are recurring, and at times, serious problems result in high mortality, morbidity, hospital visits, healthcare costs, and prolonged hospitalization. Prescription of inappropriate antimicrobial drugs during the initial stages of infection might show a high rate of mortality, even after switching to the right antimicrobial drug based on the culture-based antibiogram data. As many as $50-80 \%$ of hematological malignant patients contract various microbial infections over the course of disease and chemo/radiotherapy. This represents the exposure of a substantial number of this patient population to the risk of further mortality and morbidity. Hence, it is imperative that treating clinicians have an informed understanding of underlying immune deficiencies associated with particular malignancy, therapy regimen, clinical scenario, diagnostic outcome, and antimicrobial therapy. This could assist the clinicians in predicting the likely microbe encountered in the case and thus formulate an effective diagnostic procedure and initiate the right antimicrobial therapy.
Early diagnosis of BSIs and causative organisms facilitates the administration of empiric antibiotics which can drastically reduce secondary infection-related mortality among these patients by almost 50\% [4]. Hence, firsthand information on the microbiome of bloodstream infections could help strategize the right antimicrobial therapy for such patients. Currently, for Saudi patients of hematological malignancies, there has been no information available in the literature on the microbial load and population in BSI cases. Hence, this study was taken up to throw light on microbial population structure in BSIs among hematological malignancy patients in the Eastern province of Saudi Arabia. The study is also focused on emphasizing the role of modern NGS sequencing methods in delineating microbial population structure in hematological malignancy. Hence, this study was taken up with the objectives to screen the presence of bacterial infections in the patients of hematological malignancies on the basis of clinical evaluation and to evaluate the causative organism of bacterial infections using Direct DNA sequencing.

\section{Materials and Methods}

2.1. Study Subjects. This study was conducted in the eastern province of Saudi Arabia for two years, from March 2017 to February 2019. Patients $(n=50)$ with all types of hematological malignancies were recruited after being clinically diagnosed by an oncologist. Patients with bloodstream infections (BSI) were confirmed by consulting a hemato-oncologist, as a part of routine diagnostic follow-up. Control blood samples - with no signs of hematological malignancies $(n=50)$-were obtained from blood donors at the blood bank of the same hospital. Participants with a history of antibiotic therapy during the last two weeks were excluded from the study. All the participants filled an informed consent form and were informed about the study and study objectives. Ethical approval was obtained (No. 2016-03-164) from the institutional review board (IRB) of the Imam AbdulRahman bin Faisal University prior to the sample collection.

2.2. Sample Processing and DNA Extraction. From the recruited participants, about $5 \mathrm{ml}$ of blood samples were collected by venipuncture by a professional phlebotomist and were immediately transported to the laboratory. The blood samples were deep-frozen at $-80^{\circ} \mathrm{C}$ until further processing. The total bacterial metagenomic DNA from the collected blood was extracted using a commercially available DNA extraction kit-QIAamp cador Pathogen Mini Kit (Qiagen, Germantown, USA). The manufacturer's instructions and protocols were followed. Briefly, an aliquot of $400 \mu \mathrm{l}$ of ethylenediaminetetraacetic acid (EDTA)-anticoagulated peripheral whole blood sample was lysed with $20 \mu \mathrm{l}$ of proteinase $\mathrm{K}$ and $100 \mu \mathrm{l}$ of digestion buffer in a $2 \mathrm{ml}$ microcentrifuge tube at room temperature. The lysate containing microbial metagenome was allowed to bind to a silica column. The metagenomic DNA was eluted in $30 \mu \mathrm{l}$ of TE (Tris-EDTA) buffer which was quantified using NanoDrop UV-Vis microspectrophotometer (NanoDrop 2000, ThermoFisher Scientific, Massachusetts, USA). All the procedures were done in 
a laminar flow chamber using hydrophobic pipette filter tips to avoid external DNA contamination of the samples.

2.3. 16S rRNA Metagenomic Library Preparation. The protocols proposed by Fouhy et al. [5] were used with some modifications for the preparation of the metagenomic library. Using 16S rRNA metagenomic sequencing library protocol of Illumina, the V3 and V4 variable regions of $16 \mathrm{~S}$ ribosomal RNA bacterial gene were amplified as per standard PCR protocols. The primer pairs used for the reaction as described by Klindworth et al. [6]. Primers and overhang adapter sequences were as described by Gyarmati et al. [7] and were procured from Eurofins Genomics, Luxembourg, Belgium. The first stage PCR was run on ProFlex PCR System programmable thermocycler (ThermoFisher Scientific, Massachusetts, USA). The $25 \mu \mathrm{l}$ reaction mixture contained $2.5 \mu \mathrm{l}$ of microbial DNA $(5 \mathrm{ng} / \mu \mathrm{l}), 5.0 \mu \mathrm{l}$ each of forward primer $(1 \mu \mathrm{M})$ and reverse primer $(1 \mu \mathrm{M})$, and $12.5 \mu \mathrm{l}$ of $2 \mathrm{X}$ PCR ready mix (KAPA HiFi HotStart ReadyMix, KAPA Biosystems, Wilmington, USA). Amplification reaction condition was set at initial denaturation of $95^{\circ} \mathrm{C}$ for $3 \mathrm{mins}$ followed by 24 cycles of $0.5 \mathrm{mins}$ each of denaturation $\left(95^{\circ} \mathrm{C}\right)$, annealing of primer sets $\left(55^{\circ} \mathrm{C}\right)$, and primer-extension $\left(72^{\circ} \mathrm{C}\right)$. The reaction was kept at hold at $4^{\circ} \mathrm{C}$ after a final extension of 5 minutes at $72^{\circ} \mathrm{C}$. The PCR product thus obtained was cleaned up employing AMPureXP bead kit (Beckman-Coulter, California, USA) following manufacturer's instruction manual.

A $2^{\text {nd }}$ stage PCR was run to attach dual indices (NexteraXT Index Kit, Illumina, San Diego, USA) to the amplicons obtained in the previous step. The instructions given in the kit was followed. Briefly, PCR reaction was prepared in a $50 \mu \mathrm{l}$ reaction mixture containing $5.0 \mu \mathrm{l}$ each of Nextera XT Index primer 1 and primer 2, $25 \mu \mathrm{l}$ of PCR ready-mix, and $10 \mu \mathrm{l}$ of PCR grade water. Amplification conditions were retained the same as earlier, however, with only 8 cycles instead of 25 cycles. The PCR product thus obtained was again purified as mentioned earlier in the first stage reaction.

2.4. MiSeq Next-Generation Sequencing Run. Pooled libraries were denatured with $10 \mu \mathrm{l}$ of $0.2 \mathrm{~N} \mathrm{NaOH}$ in $1540 \mu \mathrm{l}$ of hybridization buffer (HT1) and were heat-denatured at $96^{\circ} \mathrm{C}$. An internal control PhiX was also maintained along with the sample reactions. MiSeq reagent cartridge was loaded with $5 \mu \mathrm{l}$ of $4 \mathrm{nM}$ pooled library along with equal volume of $0.2 \mathrm{~N} \mathrm{NaOH}$. The cartridge was vortexed briefly, centrifuged at $280 \times \mathrm{g}$ at room temperature for $1 \mathrm{~min}$ followed by incubation at room temperature for the dsDNA to denature to ssDNA. Finally, prechilled $990 \mu \mathrm{l}$ of HT1 butter was added to $10 \mu \mathrm{l}$ of denatured DNA and was kept ready for loading the cartridge to the MiSeq sequencing platform using $2 \times 300$ cycle V3 using the standard protocol.

2.5. Data Analysis. Secondary analysis on the system, MiSeq Reporter software was used for deducing microbiome data using comprehensive workflow mode. The metagenomic workflow was selected, which classified all the organisms based on V3 and V4 regions using the database of 16s rRNA data in the system-http://greengenes.lbl.gov/. Sequence reads $<30 \mathrm{bp}$ and quality scores $<30$ were excluded in the
Fastx toolkit. Paired-end reads are merged and filtered against the human genome build $\mathrm{Hg}$-19. Statistical analysis was performed using Mann-Whitney $U$ test with a significance of 0.05 .

\section{Results}

The present study recruited 50 hematological malignancy patients with a total of 41 bloodstream infection cases comprising of $9(21.95 \%)$ acute myeloid leukemia (5 males and 4 females), 10 (24.39\%) chronic myeloid leukemia (4 males and 6 females), 15 (36.58\%) acute lymphoid leukemia (9 males and 6 females), 5 (12.19\%) chronic lymphoid leukemia (4 males and 1 female), and 1 (2.43\%) each male patient of non-Hodgkin lymphoma and Hodgkin lymphoma. The age group ranged from 18 to 74 years with a median value of 41 years. Total leukocytes raged from 10 to 3800 cells $/ \mu l$ of blood, while neutrophils ranged from 5 to 850 cells $/ \mu l$ (Table 1). As many as 50 blood samples from healthy blood donors were considered as control samples (nonhematological malignant samples), out of which 4 individuals showed the presence of natural flora-associated Gram-positive bacteria in the sequence analysis. None of them had clinical symptoms of bloodstream infections.

\section{Sequence Reads}

MiSeq-based next-generation sequences for one hundred samples resulted in 954,546 reads assigned to bacterial operational taxonomic units (OTUs). As much as $82 \%$ of the reads were unmapped, while $16.50 \%$ of the reads were mapped to the human genome. The remaining $1.50 \%$ of the reads were mapped for different taxonomic groups of microorganisms. All 41 bloodstream infection HM patients and 4 of control samples were positive for various microbial taxa. No microbial detection occurred in non-BSI HM patients and 46 of control samples (blood donor samples).

\section{Microbial Landscape in Hematological Malignancy}

In the entire data set, the phyla Proteobacteria that includes mainly the genus of Pseudomonas and Escherichia showed the maximum population among patients of hematological malignancy and other phyla represented fewer OTUs. Firmicutes comprising of mostly Gram-positive normal flora such as Staphylococcus were more common in control samples. Groups of Actinobacteria, Fusobacteria, and Bacteroides were insignificant in both samples.

From HM patients with BSI, as many as 9 predominantly detected organisms were mapped to the database (Figure 1). An opportunistic Enterobacteriaceae family member, Escherichia coli, comprised a major chunk of the bacterial population (19.51\%), followed by Klebsiella pneumoniae (17.07\%). Coagulase-negative Staphylococcus and Viridans Streptococci accounted for $17.07 \%$ and $14.63 \%$, respectively. Other major species identified were Staphylococcus aureus (9.75\%), Pseudomonas aeruginosa (7.31\%), Acinetobacter baumannii 
TABLE 1: Characteristics of patients with hematological malignancy in this study.

\begin{tabular}{lc}
\hline Patient characteristic & Value (range, \%age) \\
\hline Median age (years) & $41(18-74)$ \\
No. (\%) of patients with diagnosis & \\
$\quad$ Acute myeloid leukemia & $9(21.95 \%)$ \\
Chronic myeloid leukemia & $10(24.39 \%)$ \\
Acute lymphatic leukemia & $15(36.58 \%)$ \\
Chronic lymphoid leukemia & $5(12.19 \%)$ \\
Non-Hodgkin lymphoma & $1(2.43 \%)$ \\
Hodgkin lymphoma & $1(2.43 \%)$ \\
WBC $(/ \mu \mathrm{l})$ & $497(8-3800)$ \\
Neutrophil $(/ \mu \mathrm{l})$ & $109(5-850)$ \\
Fever period $($ days $)$ & $4.3(1-13)$ \\
\hline
\end{tabular}

(4.87\%), Enterobacter cloacae (4.87\%), and Proteus mirabilis (4.87\%) (Figure 1).

Of the total number of reads, among the bloodstream infections in patients, $51.21 \%$ of the cases had Gramnegative infection, while control samples had no such findings. About $34.14 \%$ of the cases among patients showed Gram-positive infection, while $14.63 \%$ of cases showed polymicrobial bloodstream infections. In the case of control samples, only Gram-positive infection was found in all cases (100\%) (Figure 2). Most (90\%) of the identified reads represented opportunistic human pathogens in hematological malignancy patients, while normal human microbiota in control samples (Table 2).

\section{Types of Hematological Malignancy and Microbial Groups}

The distribution of bacterial bloodstream infections among various types of hematological malignancies in the samples is analyzed. Chronic lymphoid leukemia showed the highest percentage of both Gram-negative infections (42.86\%) and Gram-positive infections (35.71\%) than all other types of leukemias. These patients were also detected with polymicrobial infection in $50 \%$ of cases. Chronic myeloid leukemia had the second-highest rate of bloodstream infection with $33.33 \%$ Gram-negative and $28.57 \%$ of Gram-positive infection with $33.33 \%$ case rate of polymicrobial infections. Control samples (blood donors with no hematological malignancies) showed only Gram-positive infections with no signs of Gram-negative or polymicrobial infections (Figure 2).

\section{Discussion}

The present study recorded as many as 41 out of 50 samples (82\%) to be positive for a bloodstream infection among patients of hematological malignancies. This prevalence rate is similar to other studies where hematological cancer patients showed $89 \%$ BSI among the patients [8]. Nørgaard [9] opined that BSI are very common in various leukemias, indicating over $90 \%$ of patients with infection. Further, all the 41 cases of clinically diagnosed patients showed positivity for the high rate of infection in next-generation sequencing, corroborating the specificity of the modern techniques. However, though control samples had no clinically detected BSIs showing, four of the samples revealed the presence of various Gram-positive normal flora-associated microorganisms. This positivity could be attributed to the contamination of normal skin flora while venipuncture, as the organisms associated are skin-borne natural flora. Chang et al. [10] also showed that skin-associated microbial contamination of blood samples during venipuncture is common and could give false-positive reactions in blood culture and molecular methods. In a study by Jacob et al. [11], the most common hematological malignancy was acute myeloid leukemia, while our study showed that the most common ones are acute lymphatic leukemia (36.58\%). However, our small sample size could be the reason for this variation in the type of leukemias recorded.

Proteobacterial members Escherichia coli (19.51\%) and Klebsiella pneumoniae $(17.07 \%)$ were detected at a higher rate among patients of hematological malignancy. These Enterobacteriaceae family of members are best known for bloodstream infection-causing agents among individuals of compromised immunity. Several researchers have earlier showed that E. coli and Klebsiella pneumoniae are commonly isolated in patients with hematological malignancy cases [12, 13]. These opportunistic pathogens could get into the bloodstream during various medical interventions of chemo/radiotherapy procedures, as they are part of normal gut microbes.

Members of the Micrococcaceae family coagulate negative Staphylococci, and Viridans Streptococci being normal flora of the skin and oral cavity were highly detected at the rate of $17.07 \%$ and $14.63 \%$, respectively. Blijlevens et al. [14] and coworkers earlier showed that it is highly common to encounter skin-borne and oral cavity organisms to be in bloodstream infections as a result of iatrogenic transmission of the organism. Several other researchers had earlier detected coagulase-negative Staphylococci and Streptococci species in blood infections among patients of hematological malignancies [15].

Other known microbial opportunistic pathogenic groups such as Staphylococcus aureus (9.75\%), Pseudomonas aeruginosa (7.31\%), Acinetobacter baumannii (4.87\%), Enterobacter cloacae (4.87\%), and Proteus mirabilis (4.87\%) were detected at varying rates among the BSI samples of patients. Pseudomonas aeruginosa and Acinetobacter baumannii are known for drug-resistant pathogens causing serious blood infections in individuals with low immunity. Leal et al. [16] reported that these organisms were isolated from the blood cultures in case of severe bacteremia and septicemia involved in leukemia patients. Several other researchers have also found these organisms in molecular-based methods, but not in culture-based techniques [17]. Hence, this reinforces the need for molecular-based techniques like NGS in identifying pathogens in BSIs which are not covered in routine blood cultures.

Interestingly, four of the control samples with no signs of bloodstream infections showed positive reaction in sequence for coagulase-negative Staphylococci, Staphylococcus aureus, and $S$. epidermidis. However, it is uncommon to have 


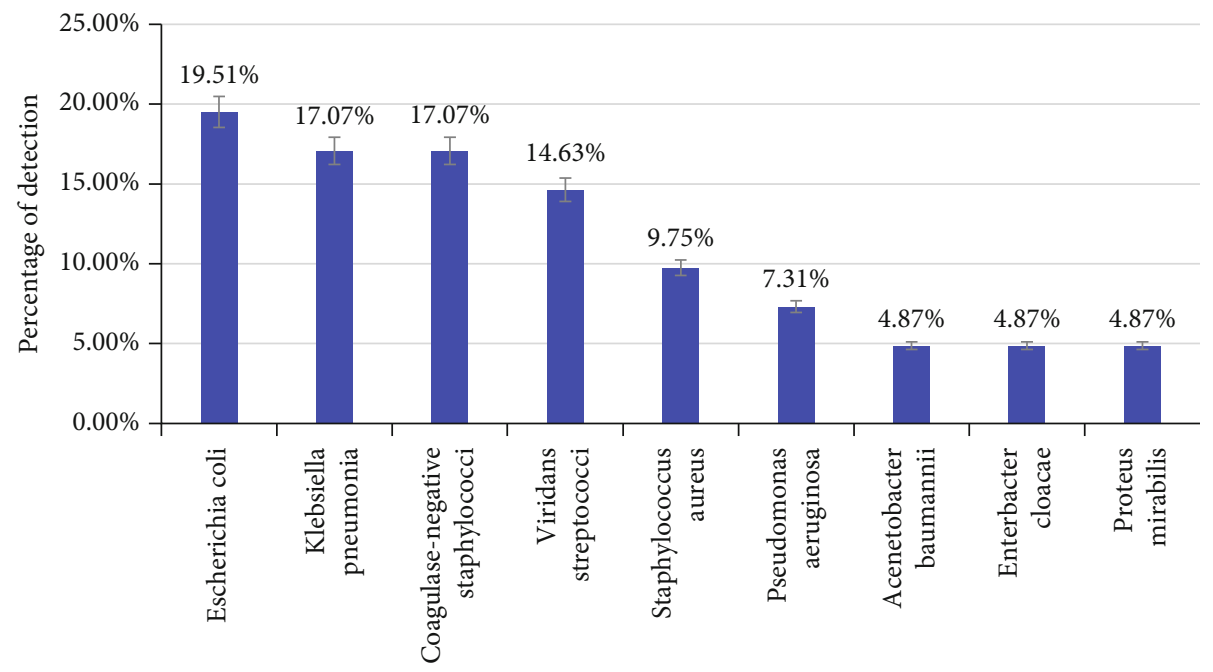

Bacterial species detected in next generation sequencing

Figure 1: Distribution of bacterial reads among hematological malignancy patients.

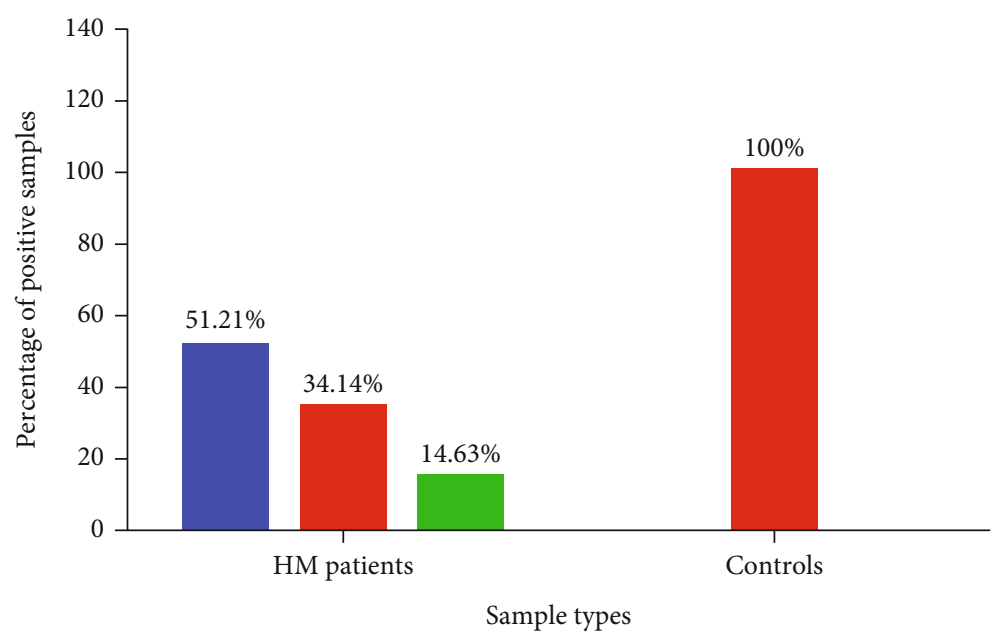

Sample types

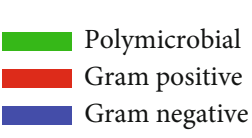

FIgURE 2: Polymicrobial bacterial infections in patients with hematological malignancies.

bloodstream infections in healthy blood donors, and the population structure of the microorganism indicates a possible skin microflora contamination during venipuncture, as these organisms are common microflora of the skin. Hall and Lyman [17] had earlier reported the contamination of skin microflora in case of blood culture and other molecular techniques. Hence, it is unlikely that these microorganisms had resulted in bloodstream infections in such blood donors. Further, history records of the blood donors state no signs and symptoms of any infections in the previous fifteen days and hence could be attributed to venipuncture artefacts.

Of the total number of reads, among bloodstream infections in patients, $51.21 \%$ of the cases had Gram-negative infection, while control samples had no such findings. About $34.14 \%$ of the cases among patients showed Gram-positive infection, while $14.63 \%$ of the cases showed polymicrobial bloodstream infections. In the case of control samples, only Gram-positive infection was found in all cases (100\%) (Figure 2). Most (90\%) of the identified reads represented opportunistic human pathogens in HM patients, while there was normal human microbiota in control samples. Trecarichi et al. [12] had earlier reported that BSI are mainly caused by the Gram-negative group of microorganisms and more so in cancer patients. Their study showed the most frequently isolated Gram-negative bacteria were E. coli and K. pneumoniae, which is in line with our studies, wherein E. coli was $19.51 \%$ and K. pneumoniae was $17.07 \%$ and were the most frequently isolated Gram-negative bacteria. Generally, the Gramnegative members are more virulent in causing infections in humans; hence, they are more frequently isolated. However, 
TABLE 2: Bacterial species detected in blood samples from hematological malignancies.

\begin{tabular}{|c|c|c|c|c|}
\hline Hematologic malignancies $(n=50)$ & No. (\%) & Control $(n=50)$ & No. $(\%)$ & $p$ value \\
\hline Blood stream infection & $41(82)$ & Blood stream infection & $04(8.0)$ & $<0.05$ \\
\hline Gram-negative group & $24(58.53)$ & Gram-negative group & & \\
\hline Escherichia coli & $08(19.51)$ & - & - & $<0.05$ \\
\hline Klebsiella pneumoniae & $07(17.07)$ & - & - & $<0.05$ \\
\hline Pseudomonas aeruginosa & $03(7.31)$ & - & - & $<0.05$ \\
\hline Enterobacter cloacae & $02(4.87)$ & - & - & NS \\
\hline Acinetobacter baumannii & $02(4.87)$ & - & - & NS \\
\hline Proteus mirabilis & $02(4.87)$ & - & - & NS \\
\hline Gram-positive group & $17(43.5)$ & Gram-positive group & & \\
\hline Coagulase -ve staphylococci & $07(17.07)$ & Coagulase -ve staphylococci & $01(2.0)$ & NS \\
\hline Staphylococcus aureus & $04(9.75)$ & Staphylococcus aureus & $01(2.0)$ & NS \\
\hline Viridians streptococci & $06(14.63)$ & Staphylococcus epidermidis & $02(4.0)$ & NS \\
\hline
\end{tabular}

NS: nonsignificant; $p<0.05$ is statistically significant.

infection with both Gram-negative and Gram-positive organisms (polymicrobial) is also noted in the case of Trecarichi et al. [12]. Similar to these results, the present study also documents polymicrobial infections in bloodstream infections of the patients studied, making it more complex for treatment and management. However, control samples showed no polymicrobial infection, instead only Grampositives-indicating possible contamination-associated microorganisms.

Chronic lymphoid leukemia showed the highest percentage of both Gram-negative infections (42.86\%) and Grampositive infections $(35.71 \%)$ than all other types of leukemias. These patients were also detected with polymicrobial infection in $50 \%$ of cases, indicating the complexity of the bloodstream infections in leukemia patients and difficulty in its antimicrobial management. Andersen et al. [18] and coworkers showed that CLL patients are more prone to bloodstream infections owing to the severity of the disease and therapeutic options available. Several other workers also reported a high rate of bloodstream infections in CLL patients [18, 19]. Chronic myeloid leukemia had the second-highest rate of bloodstream infection with $33.33 \%$ Gram-negative and $28.57 \%$ of Gram-positive infection with $33.33 \%$ case rate of polymicrobial infections. Control samples (blood donors with no hematological malignancies) showed only Gram-positive infections with no signs of Gram-negative or polymicrobial infections (Figure 2). This indicates the possibility of contamination of the blood samples during venipuncture and the results not attributed to blood infections.

In recent years, there has been an increase in the incidence rates of hematological malignancies, particularly among the Central Province, Eastern region, and Northern region populations [20]. Hematological malignancies, by virtue of their treatment modalities, put the patients at higher risk of microbial infections. A broad range of infectious agents-bacteria, fungi, protozoa, and viruses-can infect locally or systemically. Unscrupulous use of a wide array of antibiotics for prophylaxis in cancer patients has led to the development of drug-resistance among many bacterial spe- cies. Controlling immune deficit, preventing microbial infections, and implementing antibiotic stewardship are some of the key aspects of the management of hematological malignant patients. For favorable outcomes, accurate diagnosis of the infection and timely initiation of appropriate antibiotic therapy are imperative. Hence, NGS-based detection methods can pave the way for the detection of BSIs in such patients timely and specifically.

\section{Conclusions}

The present study detects that there exists a high rate $(82 \%)$ of bloodstream infections among patients of hematological malignancies in Saudi Arabia. Gram-negative opportunistic, Proteobacteria group members such as Escherichia coli, Klebsiella pneumoniae, and Pseudomonas aeruginosa are highly detected in these cases, while normal blood donors with no hematological malignancies showed insignificant microbial population. Most of the bloodstream infections in patients were characterized by polymicrobial infections unlike control in samples, indicating the complexity of the infection and subsequent antimicrobial therapy. Next-generation sequencing showed robust, fast, and specific identification of infectious agents in bloodstream infections in hematological malignancies, indicating the possibility of its application in routine follow-up of such patients for infections. However, the limitations of this study, such as low sample size and restricted geographic location of the sampling, may be taken into account when further research work is designed to include more samples with representation from all the geographic regions. Future research is required on the study of molecular characterization of multidrug resistance among bloodstream infections causing microorganisms identified in such patients using next-generation sequencing.

\section{Data Availability}

The data used to support the findings of this study are included within the article. 


\section{Consent}

A total of 50 patients and 50 healthy subjects were recruited into the study, and all the participants were provided with informed consent forms to ensure the confidentiality of the data collected from them.

\section{Conflicts of Interest}

The authors declare that there are no conflicts of interest regarding the publication of this paper.

\section{Acknowledgments}

The authors are very thankful to the Deanship of Scientific Research, Imam Abdulrahman Bin Faisal University, Dammam, for fully funding this project.

\section{References}

[1] D. Rodriguez-Abreu, A. Bordoni, and E. Zucca, "Epidemiology of hematological malignancies," Annals of Oncology, vol. 18, no. 1, pp. i3-i8, 2007.

[2] A. Bawazir, N. Al-Zamel, A. Amen, M. A. Akiel, N. M. Alhawiti, and A. Alshehri, "The burden of leukemia in the Kingdom of Saudi Arabia: 15 years period (1999-2013)," BMC Cancer, vol. 19, no. 1, p. 703, 2019.

[3] F. E. Al-Otaibi, E. E. Bukhari, M. Badr, and A. A. Alrabiaa, "Prevalence and risk factors of Gram-negative bacilli causing blood stream infection in patients with malignancy," Saudi Medical Journal, vol. 37, no. 9, pp. 979-984, 2016.

[4] P. Wadhwa and V. Morrison, "Infectious complications of chronic lymphocytic leukemia," Seminars in Oncology, vol. 33, no. 2, pp. 240-249, 2006.

[5] F. Fouhy, J. Deane, M. C. Rea et al., "The effects of freezing on faecal microbiota as determined using MiSeq sequencing and culture-based investigations," PLoS One, vol. 10, no. 3, article e0119355, 2015.

[6] A. Klindworth, E. Pruesse, T. Schweer et al., "Evaluation of general 16S ribosomal RNA gene PCR primers for classical and next-generation sequencing-based diversity studies," Nucleic Acids Research, vol. 41, no. 1, article e1, 2013.

[7] P. Gyarmati, C. Kjellander, C. Aust, M. Kalin, L. Öhrmalm, and C. G. Giske, "Bacterial landscape of bloodstream infections in neutropenic patients via high throughput sequencing," PLoS One, vol. 10, no. 8, article e0135756, 2015.

[8] H. Gedik, F. Simşek, A. Kantürk et al., "Bloodstream infections in patients with hematological malignancies: which is more fatal - cancer or resistant pathogens?," Therapeutics and Clinical Risk Management, vol. 10, pp. 743-752, 2014.

[9] M. Nørgaard, "Risk of infections in adult patients with haematological malignancies," The Open Infectious Diseases Journal, vol. 6, no. 1, pp. 46-51, 2012.

[10] C. J. Chang, C. J. Wu, H. C. Hsu et al., "Factors associated with blood culture contamination in the emergency department: critical illness, end-stage renal disease, and old age," PLoS One, vol. 10, no. 10, article e0137653, 2015.

[11] L. A. Jacob, K. C. Lakshmaiah, K. Govindbabu et al., "Clinical and microbiological profile of febrile neutropenia in solid tumors and hematological malignancies at a tertiary cancer care center in South India," Indian Journal of Cancer, vol. 51, no. 4, pp. 464-468, 2014.

[12] E. M. Trecarichi, L. Pagano, A. Candoni et al., "Current epidemiology and antimicrobial resistance data for bacterial bloodstream infections in patients with hematologic malignancies: an Italian multicentre prospective survey," Clinical Microbiology and Infection, vol. 21, no. 4, pp. 337-343, 2015.

[13] Ö. Kara, P. Zarakolu, S. Aşçioğlu et al., "Epidemiology and emerging resistance in bacterial bloodstream infections in patients with hematologic malignancies," Infectious Diseases, vol. 47 , no. 10, pp. 686-693, 2015.

[14] N. M. Blijlevens, J. P. Donnelly, and B. E. de Pauw, "Empirical therapy of febrile neutropenic patients with mucositis: challenge of risk-based therapy," Clinical Microbiology and Infection, vol. 7, no. 4, pp. 47-52, 2001.

[15] W. Krüger, B. Rüssmann, N. Kröger et al., "Early infections in patients undergoing bone marrow or blood stem cell transplantation - a 7 year single centre investigation of 409 cases," Bone Marrow Transplantation, vol. 23, no. 6, pp. 589-597, 1999.

[16] H. F. Leal, J. Azevedo, G. E. O. Silva et al., "Bloodstream infections caused by multidrug-resistant gram-negative bacteria: epidemiological, clinical and microbiological features," $B M C$ Infectious Diseases, vol. 19, no. 1, p. 609, 2019.

[17] K. K. Hall and J. A. Lyman, "Updated review of blood culture contamination," Clinical Microbiology Reviews, vol. 19, no. 4, pp. 788-802, 2006.

[18] M. A. Andersen, C. E. Moser, J. Lundgren, and C. U. Niemann, "Epidemiology of bloodstream infections in patients with chronic lymphocytic leukemia: a longitudinal nation-wide cohort study," Leukemia, vol. 33, no. 3, pp. 662-670, 2019.

[19] C. Kjellander, M. Björkholm, O. Källman et al., "Bloodstream infections in patients with chronic lymphocytic leukemia: a longitudinal single-center study," Annals of Hematology, vol. 95, no. 6, pp. 871-879, 2016.

[20] I. G. Alghamdi, I. I. Hussain, M. S. Alghamdi, A. A. Dohal, and M. A. el-Sheemy, "The incidence of leukemia in Saudi Arabia. Descriptive epidemiological analysis of data from the Saudi Cancer Registry 2001-2008," Saudi Medical Journal, vol. 35, no. 7, pp. 674-683, 2014. 